\title{
Simulating Fluorescence Light-Canopy Interaction in Support of Laser-Induced Fluorescence Measurements
}

\section{A. Rosema}

EARS Ltd Remote Sensing Consultants, Delft, The Netherlands

\section{W. Verhoef}

National Aerospace Laboratory, Remote Sensing Department, Emmeloord, The Netherlands

\section{J. Schroote and J. F. H. Snel}

Department of plant Physiological Research, Agricultural University of Wageningen, The Netherlands

In the Netherlands an operational field instrument for the measurement of laser induced fluorescence of vegetation (LEAF) is developed. In addition, plant physiological and remote sensing research is done to support this new remote sensing instrument. This paper presents a general introduction on the subject of laser-induced fluorescence, including the relation between chlorophyll fluorescence and photosynthesis, spectral characteristics, and previous research. Also the LEAF system is briefly described. Subsequently, the development of a leaf fluorescence model (KMF) and a canopy fluorescence model (FLSAIL) are reported. With these simulation models a sensitivity study is carried out. Fluorescence of $685 \mathrm{~nm}$ appears to be most suitable to obtain information on photosynthesis and stress, but is also influenced by canopy structure. Separation of these two effects is studied.

Address correspondence to A. Rosema, EARS Ltd. Remote Sensing Consultants, P.O. Box 449, 2628 EB, Delft, The Netherlands. Received 28 December 1990; revised 14 April 1991.

\section{INTRODUCTION}

The eighties have been a decade of growing awareness of the vulnerability of our environment, and this has been the line of action for many scientists in a wide range of disciplines. For several years we have addressed the question of the possibility of detecting plant stress due to soil and air pollution by means of remote sensing techniques. Chlorophyll fluorescence, which is a signal related to the performance of the plant photosynthetic apparatus, seemed a most promising approach. In 1987 the LIFT project (Laser Induced Fluorescence in Trees) was carried out, a cooperative Dutch-Italian effort (Rosema et al., 1988). Monitoring a Douglas fir with the FLIDAR laser fluorescence system (Castagnoli et al., 1986), chlorophyll fluorescence was found to exhibit dramatic changes during the day. These changes were shown to be correlated to air pollution levels of ozone and nitrogen oxides and could be partly explained in terms of effects on photosynthesis.

These results have contributed to a growing enthusiasm for this technique and have set the green light for further research. In the Nether- 
lands the LEAF project was approved and financed by the Netherlands Remote Sensing Board in 1989. This project consists of two parts: instrument development and application research. The Laser Environmental Active Fluorosensor (LEAF) is a field instrument dedicated to the fast and user-friendly collection of remotely sensed fluorescence and reflection data in four spectral bands. This instrument will be commercially available and will allow a larger part of the remote sensing community to enter this challenging area.

The research part of the LEAF project consists of two components: 1) plant physiological research and 2) remote sensing research. Plant physiologists were surprised by the unexpected large range of fluorescence values measured during the LIFT project. For this reason they investigate the relation between short laser pulse induced fluorescence and the "pulse amplitude modulation" (PAM) technique (Schreiber et al., 1986) on plants subjected to air pollution and other stress factors. The PAM technique is a contact measurement, widely used in photosynthesis research, but not suitable for remote sensing.

The remote sensing component of the LEAF research project focuses on the methodology of measuring and interpretation. Canopy fluorescence is not only dependent on the rate of photosynthesis; due to reabsorption and scattering, it is also influenced by the properties of leaf and canopy, particularly chlorophyll concentration, leaf area index, and leaf angle distribution. It is our objective to separate between these effects and to extract the information on photosynthesis. For this purpose we have developed a fluorescence light-canopy interaction model, which is the main subject of our present paper. However, before treating our modeling effort, including a sensitivity study, a general introduction will be presented in this new field in remote sensing.

\subsection{Chlorophyll Fluorescence and Photosynthesis}

Photosynthesis is taking place inside the chloroplasts of the plant cell (Fig. 1). It is a light driven reaction with the final result that carbon dioxide and water are converted into glucose and oxygen. The reaction consists of a complex reductionoxidation chain, along which electrons are transported from water to $\mathrm{CO}_{2}$. At one end of the chain, water is oxidized and oxygen generated,

$$
2 \mathrm{H}_{2} \mathrm{O} \rightarrow 4 \mathrm{H}^{+}+\mathrm{O}_{2}+4 e^{-},
$$

while at the other end carbon dioxide is reduced to glucose,

$$
\mathrm{CO}_{2}+4 \mathrm{H}^{+}+4 e^{-} \rightarrow 2 \mathrm{CH}_{2} \mathrm{O}
$$

The electron transport between the two ends of the reaction chain is driven by small electron "pumps," called photosystems. There are two kinds of photosystems that work serially together in transporting electrons: PS2 and PS1. These are chlorophyll-protein complexes, embedded in the "thylakoid," a membrane of lipids and proteins inside the chloroplast (Fig. 2). The photosystems absorb light and in this way become electronically excited. "Excitons" travel randomly through the

Figure 1. Photosynthesis takes place at the grana thylakoid membrane, inside the chloroplast (Amesz and Vredenberg, 1982).

\section{CHLOROPLAST}

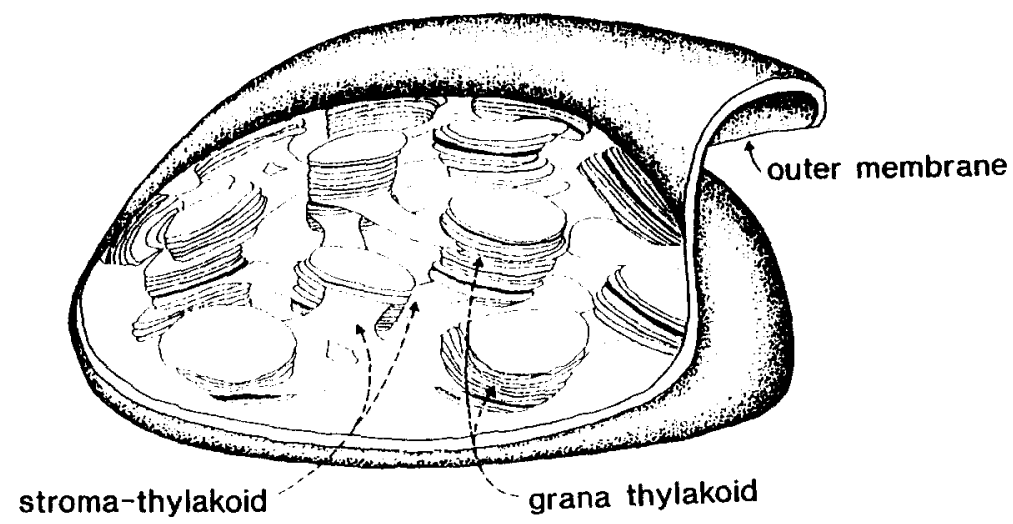


photosystems and may loose their excess energy by several, competing mechanisms. Each photosystem has a reaction center where the electron may be trapped and passed to an acceptor molecule, thus driving the photosynthetic electron transport chain. Other possibilities of deexcitation are chlorophyll fluorescence and heat conversion. If an exciton arrives at the reaction center, while the acceptor is still reduced ("closed"), then it will continue its path through the photosystem and will probably lose its energy by one of the competing mechanisms. The distribution of light energy can be illustrated by the following generalized example. Under optimal conditions and relatively low light levels, the use of absorbed light quanta would be approximately as follows:

Photosynthesis: $84 \%$

Heat: $14 \%$

Fluorescence: $2 \%$

However, if photosynthesis is completely blocked, for example, by a herbicide like DCMU, all excitation energy is converted to heat and fluorescence, and this may result in the following distribution:

Photosynthesis: $0 \%$

Heat: $88 \%$

Fluorescence: $12 \%$

Thus a sixfold increase of the chlorophyll fluorescence is possible in the most extreme case. At higher light levels photosynthesis approaches saturation. The plant cannot use the excess light energy and has mechanisms to convert the excess energy into heat. In general, however, it can be concluded that relatively high fluorescence indicates impeded photosynthesis and consequently plant stress.

For more extended information on chlorophyll fluorescence and its applications the interested reader is referred to the Proceedings of the International Chlorophyll Fluorescence Symposium (Lichtenthaler, 1988).

\subsection{Fluorescence Spectral Characteristics}

The photosystem excitation is spectrally most sensitive to blue and red light, corresponding to the

Figure 2. Sketch of the simplified structure of the thylakoid membrane, consisting of a lipid double layer with embedded photosystems and protein complexes, which are involved in photosynthetic electron transport (Amesz and Vredenberg, 1982).

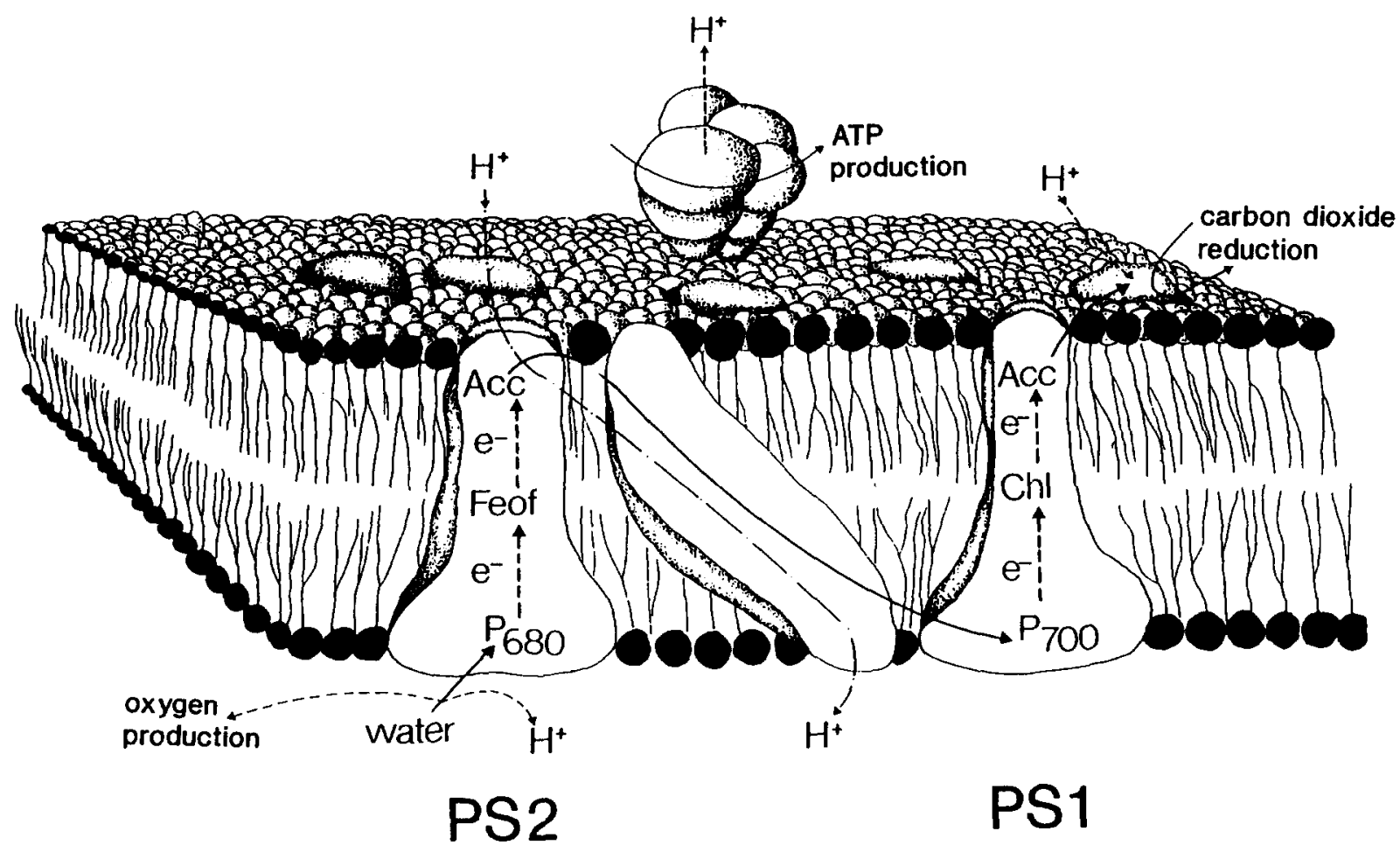




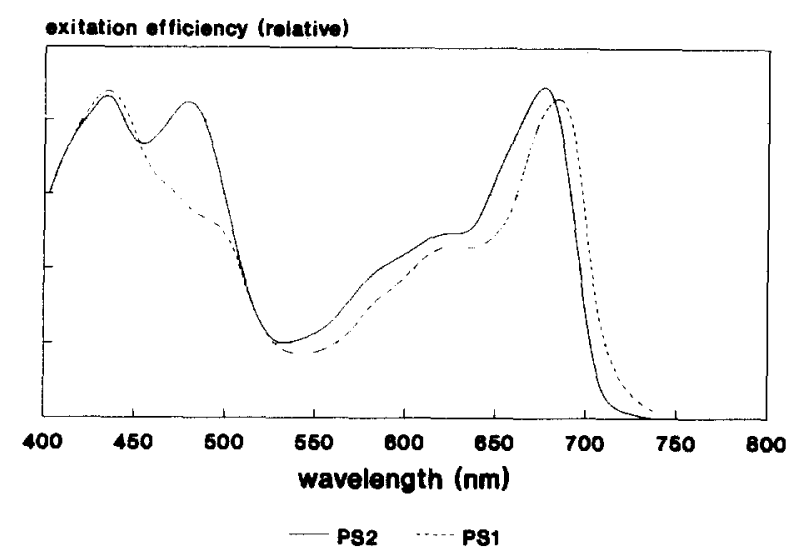

Figure 3. The relative excitation efficiency of light for photosystem 2 and photosystem 1 (Barber et al., 1982).

two absorption maxima, peaking at $435 \mathrm{~nm}$ and $670 \mathrm{~nm}$ (Fig. 3). Although absorption is minimal in the green at about $540 \mathrm{~nm}$, still about $45 \%$ of the quanta is absorbed. The energy required to transfer an electron to the acceptor of PS2 corresponds to that of a red photon around $675 \mathrm{~nm}$. The extra energy of shorter wavelength photons is dissipated into heat.

Under natural light conditions, the fluorescence spectrum of green vegetation extends in the red and near infrared, roughly between $660 \mathrm{~nm}$ and $780 \mathrm{~nm}$. By means of UV lasers, fluorescence may also be excited in the blue (Chapelle et al., 1985). This blue fluorescence, of which the plant physiological meaning is not yet understood, is outside the scope of this paper. In the red and near infrared part of the spectrum two partly overlapping fluorescence bands are observed, with maxima at $685 \mathrm{~nm}$ and $730 \mathrm{~nm}$ (Fig. 4), corresponding to electronic decay from the first excited

Figure 4. Fluorescence spectrum induced with laser excitation at $480 \mathrm{~nm}$.



singlet state to the lowest and first vibrational level of the ground state. Plant physiologists disagree on the question whether PS1 contributes significantly to the fluorescent emission. Holzwarth (1988), however, studied fluorescence spectra associated with distinct fluorescence decays, and showed for pea that PS2 fluorescence dominated at $685 \mathrm{~nm}$ and PS1 fluorescence at $730 \mathrm{~nm}$. PSI fluorescence, however, is considered to be invariable; that is, it would not be effected by changes in efficiency of the photosynthesis process.

When measuring the stationary fluorescence of various leafs, considerable differences in the ratio of the 730 and 685 emission may be observed. These, however, cannot simply be interpreted in terms of fluorescence yield changes at the photosystem level. The fluorescence emitted by the photosystems is, of course, subject to scattering and reabsorption. This implies that the fluorescence measured at the leaf or canopy level depends also on chlorophyll density, leaf thickness, canopy architecture, and background reflection. In this respect there is a strong difference between the $685 \mathrm{~nm}$ and the $730 \mathrm{~nm}$ band. Although reabsorption is very strong and scattering relatively weak at $685 \mathrm{~nm}$, the opposite is the case at 730 $\mathrm{nm}$. The consequence is an enhancement of 730 $\mathrm{nm}$ fluorescence, relative to $685 \mathrm{~nm}$, with the effect that in canopy spectra the heights of the two peaks are of the same order of magnitude, whereas in some cases the $730 \mathrm{~nm}$ peak may even be higher.

\subsection{Previous Research on Remote Sensing of Plant Fluorescence}

The application of chlorophyll fluorescence in remote sensing has been studied for some time by a limited number of scientists. Possibly the earliest dedicated instrument for measuring fluorescence is the Fraunhofer Line Discriminator, although the position of the Fraunhofer line in question $(656.3 \mathrm{~nm})$ is far from optimal to study chlorophyll fluorescence. Watson et al. $(1974 ; 1976)$ observed enhanced fluorescence of Pinus growing on soil with high $\mathrm{Cu}$ and $\mathrm{Zn}$ content. Using the same instrument on Citrus trees, MacFarlane et al. (1980) found a high response to water stress.

Another passive approach is to try to extract fluorescence from reflection data, on which it is superimposed. This was shown possible when applied to algae (Neville and Gower, 1976; Kim 
et al., 1985). Rosema and Werner (1983) investigated this approach for land vegetation, using a simulation model. They found that fluorescence could notably modify the (apparent) reflection and tentatively proposed the use of a fluorescence index based on the reflection difference in two narrow bands at $650 \mathrm{~nm}$ and $690 \mathrm{~nm}$.

Laser-induced fluorescence (LIF) is an active measuring technique that seems most promising. Early work was mainly related to sea applications (Hickman et al., 1972; Hoge and Swift, 1980; 1981; 1983; Gehlhaar et al., 1981; Castagnoli et al., 1986). Application to land vegetation followed (MacFarlane et al., 1980; Hoge et al., 1983; Cecchi et al., 1984). Chapelle et al. (1984a, b; 1985) studied LIF in relation to water stress, chlorosis, nutrient deficiencies, and plant type. Using UV excitation, they also found fluorescence bands at $440 \mathrm{~nm}$ and $525 \mathrm{~nm}$. Finally, Rosema et al. (1988) used the fluorescence lidar of IROECNR (Castagnoli et al., 1986) on Douglas fir in the field and demonstrated effects of air pollution on photosynthesis.

\subsection{Previous Research on (Fluorescence) Light-Canopy Interaction}

Allen and Richardson (1968) published a model to describe the reflection and transmission of leaves on the basis of the Kubelka-Munk theory of radiation transfer in diffuse scattering media. Fukshansky and Kazarinova (1980) extended the theory to fluorescent media, and found an approximate analytical solution for a plant leaf. Rosema and Werner (1983) developed a numerical leaf model on the basis of the extended theory and investigated the contribution of fluorescence to the "apparent" reflection. It was shown that, in the red, fluorescence may have a significant contribution.

The most widely used line of canopy models in remote sensing is that of Suits (1972), Bunnik (1978), and Verhoef (1984). They are originally based on Duntley's extension of KM theory, incorporating a direct solar flux, besides a diffuse flux. If we want to apply chlorophyll fluorescence techniques at the canopy level, then it is clear that we have to include fluorescence in these models as well. This has been done in the present project by combining the SAIL model of Verhoef (1984) and the KMF leaf model, which was originally developed by Rosema and Werner (1983).

\subsection{The LEAF Instrument}

The LEAF (Laser Environmental Active Fluorosensor) instrument is a new remote sensing fluorosensor, developed in the present project and intended for operational use. It uses a laser pulse as a probe and detects radiation, either reflected or fluorescence, in four wavelength bands. A diagram of the LEAF instrument is presented in Figure 5. A Nd-Yag laser produces $10 \mathrm{~mJ}$ pulses of $10 \mathrm{~ns}$ width at a wavelength of $532 \mathrm{~nm}$. The laser pulse is diverged to give an adjustable field of view of 5-20 mrad. The field of view of the laser optics coincides with the field of view of the detection optics. Radiation will be detected during a time window to suppress the background signal. The position of the time window depends on the distance to the canopy. It is set automatically by focusing the object lens. The width of the time window depends on the pulse duration, the "decay" time of the fluorescence, the depth of the measured canopy and the uncertainty in the canopy distance. It can be chosen by the user. Radiation is collected by a telescope and deviated to four photomultipliers by means of beamsplitters. Each photomultiplier has its own optical filter for selecting different wavelength bands. In the present setup the measured wavelength bands are:

Band 1: $640-660 \mathrm{~nm}$
Band 2: $675-695 \mathrm{~nm}$
Band 3: 720-740 nm
Band 4: 770-800 nm

The laser-induced fluorescence response is obtained by subtracting two measurements: one with laser action (active) and one without (passive). Fluorescence occurs mainly in Bands 2 and 3. Bands 1 and 4 were chosen to allow the determination of a passive fluorescence index and a vegetation index (see Section 2.3). However, in principle all bands allow the measurement of reflection (passive) and fluorescence (active). Other wavelength bands may be chosen by changing the filters.

The operation of the LEAF instrument is designed to be user-friendly. The instrument's action is controlled by a laptop 80386 field computer. The field of view is selected and photographed by means of an adapted camera. The width of the time window and the frequency of measurements can be specified by the user. The repetition rate is between $2 \mathrm{~Hz}$ and one shot per hour. Accuracy 


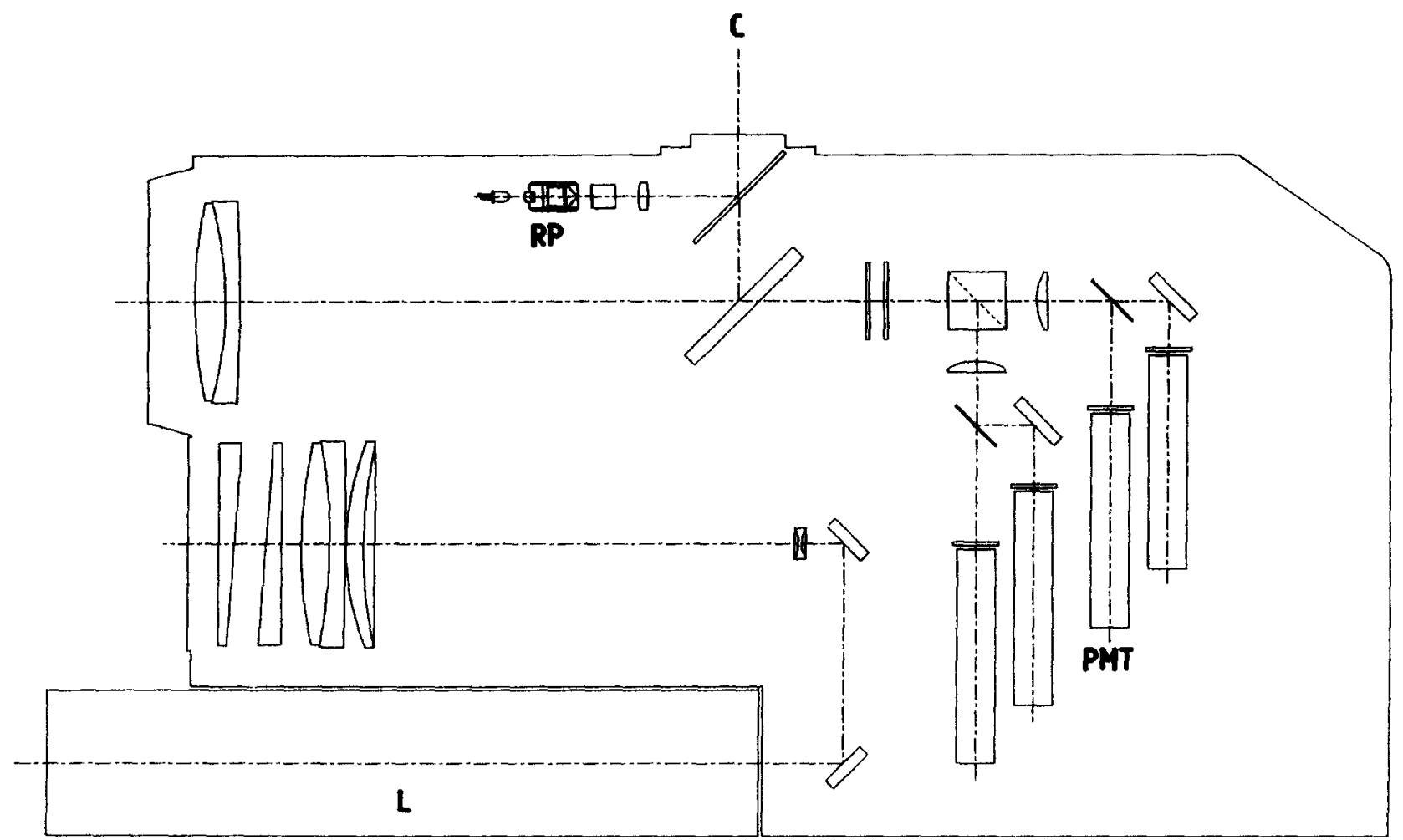

Figure 5. Impression of the Laser Environmental Active Fluorosensor (LEAF).

will be better than $1 \%$. Measurements can be taken both in the lab ( $3 \mathrm{~m}$ distance, $15 \mathrm{~mm}$ spot size) and in the field (distance $10-100 \mathrm{~m}$, spotsize 0.5 at $10 \mathrm{~m}$ distance).

\section{MODELING FLUORESCENCE LIGHT-CANOPY INTERACTION}

The fluorescence of plants at the canopy level is not only determined by the efficiency of plant photosynthesis, but also by chlorophyll density and canopy architecture. Simulation models, describing the complex interaction between the crop, the excitation light, and the fluorescence, may help to understand the relative significance of the various parameters and to develop strategies to separate the information on photosynthesis from other effects. Here it is noted that the other canopy properties are not without interest, but they may as well be investigated by passive (reflection) techniques. In fact, since LIF measurements generally imply crop radiance measurements with and without laser pulse, we will also consider the possible use of the passive measurement for estimating and separating the canopy effect.

\subsection{The Kubelka-Munk Fluorescence Model (KMF)}

This model applies to radiation transport in homogeneous, diffusely scattering media with absorption and fluorescent emission. It is based on the following modification of the original KubelkaMunk differential equations

$$
\begin{aligned}
d F^{+} / d z & =-(k+s) F^{+}+s F^{-}+0.5 P, \\
-d F^{-} / d z & =-(k+s) F^{--}+s F^{+}+0.5 P .
\end{aligned}
$$

Here $F^{+}$is the upward and $F^{-}$the downward fluorescent radiation flux density. $k$ and $s$ are the Kubelka-Munk absorbtion and backscatter coefficient, respectively. The spectral fluorescent emission (or production) term $(P)$ is calculated in the following way:

$$
P=\phi \eta \int_{\lambda_{2}}^{\lambda_{1}} k\left(E^{+}+E^{-}\right)\left(\lambda / \lambda_{670}\right) d \lambda .
$$

This equation states that all absorbed photons of the upward and downward excitation flux $\left(E^{+}, E^{-}\right)$, in the excitation wavelength range $\lambda_{1}-\lambda_{2}$, contribute to the excitation of the photosystems and that a fraction $\phi$ gives rise to fluorescence. $\phi$ is the photon fluorescence efficiency. The fluorescent emission has a given spectral distribution $(\eta)$, which is assumed to be constant and 
known. Of course, in the case of monospectral excitation (with a laser), the above integration is not necessary.

Fukshansky and Kazarinova (1980) solved the above system of Eqs. (3)-(5) for $k$ and $s$ by approximation in an analytical way, given the measured leaf reflection and transmission. Rosema and Werner (1983) assumed $k$ and $s$ to be given and solved for the upward and downward fluxes $\left(E^{+}, F^{+}\right.$and $\left.E^{-}, F^{-}\right)$in a numerical way, thus obtaining the leaf reflection, transmission, and front and back side fluorescence for given optical properties.

The numerical solution of the above system was originally obtained by means of an implicit finite difference scheme. This time we applied the so-called "doubling method." Both methods give the same result. The latter, however, is faster, considered more elegant, and for this reason preferred. The doubling method is explained in the Appendix.

\subsection{The Canopy Reflectance and Fluorescence Model (FLSAIL)}

The SAIL canopy reflectance model, Verhoef (1984), is a refinement of the first bidirectional reflectance model for vegetation canopies, developed by Suits (1972). The refinement concerns the incorporation of a leaf inclination distribution function (LIDF), which allows us to obtain more realistic angular characteristics of bidirectional reflectance. In both models radiative transfer inside a canopy layer is described by means of a system of four linear differential equations, which contain nine coefficients that can be expressed as functions of leaf transmittance, leaf reflectance, LIDF, leaf area index (LAI), solar zenith angle, viewing zenith angle, and relative azimuth angle. It describes absorption and scattering of monospectral radiant fluxes, which means that it is not applicable to the phenomenon of fluorescence, since this implies the conversion of shortwave excitation light into longer wavelength fluorescence. Therefore, fluorescence in vegetation canopies should be modeled as a bispectral system of eight differential equations. This is the approach taken.

A traditional manner of solving such a system of linear differential equations is by eigenvector analysis (Verhoef, 1985). However, this analytical method becomes rapidly more tedious with increasing dimension of the system. For this reason a numerical solution, by means of the "doubling method," was prefered. This method is fast, elegant, and accurate. As a result, it gives the bulk optical properties of a layer of vegetation, that is, the reflectances, transmittances, and fluorescence rates, associated with the incident and scattered flux. Finally these are combined with the reflectances of the underlying soil by means of the "adding" method (Verhoef 1985), in order to compute the reflectances and the fluorescence of the ensemble.

An extension introduced in the SAIL model in 1989 (Verhoef, forthcoming) is the incorporation of the "hot spot" effect. This effect occurs when the viewing direction approaches the direction of the sun and consists of an enhanced reflectance due to the fact that the fraction of observed shadow area tends to disappear in this region. In the center of the hot spot, both directions coincide and only sunlit leaves and sunlit soil are observed, which explains the relatively high reflectance. The hot spot effect has been included in the SAIL model in a manner suggested by Kuusk (1985). It requires the specification of one extra input parameter, namely, the hot spot size parameter, defined as the ratio of the horizontal correlation length $(l)$ and canopy height $(h)$, where $l$ is to be estimated from leaf size. Since this effect cannot be described by traditional radiative transfer formulations, it is included as a separate calculation of two single scattering contributions and one "single fluorescence" contribution.

The present incorporation of fluorescence into the SAIL model has resulted in a new model, called FLSAIL. Extinction and scattering at the excitation wavelength and at the fluorescence wavelength are treated in exactly the same way as in the original SAIL model. Fluorescence from single leaves is supposed to take place similarly to reflection and transmission in the monospectral case. It is assumed that total fluorescence is proportional to the flux incident on the leaf at the excitation wavelength, that it is divided in a backward and a forward fraction, and that it is perfectly diffuse. In this case the fluorescence coefficients of the bispectral system of differential equations are similar to the corresponding scattering coefficients of the original SAIL model, if single leaf reflectance and transmittance are replaced by backward and forward fluorescence, respectively.

A novel approach in the FLSAIL model is that the optical properties of the leaves are not entered 


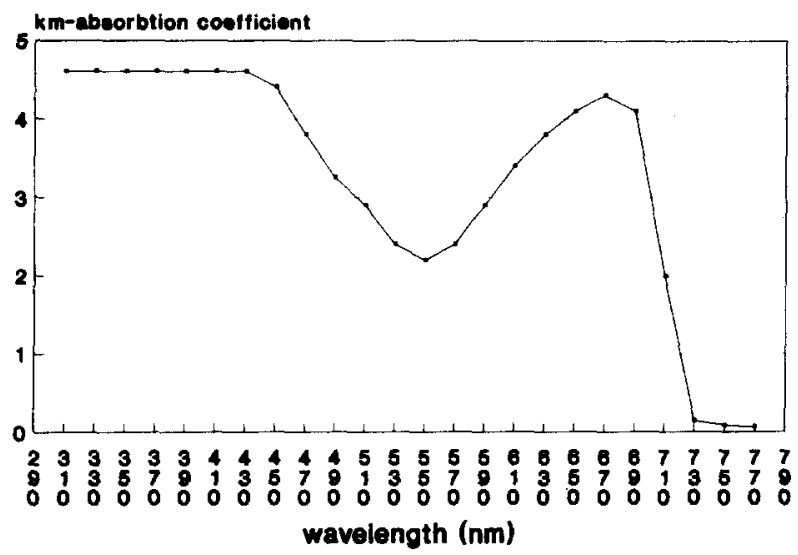

Figure 6. Spectral distribution of the KM absorption coefficient $(k)$ as used in the KMF model.

as input data to the model, but are computed with the KMF single leaf fluorescence model. This submodel, discussed in the previous section, also uses the doubling method in order to compute reflectance, transmittance, and front and backside fluorescence of the leaf.

The complete numerical solution of the FLSAIL equations by means of the doubling method is quite extensive and will not be discussed here. The method is explained in principle for the case of the single leaf in the Appendix.

\subsection{Model Data}

The two simulation models use data on leaf optical properties, depending on wavelength. In general, these data, specified for each $20 \mathrm{~nm}$ interval in the wavelength region $300-780 \mathrm{~nm}$, are derived from the literature. The spectral distribution of the absorption coefficient $(k)$ and backscatter coefficient $(s)$ were derived from reflectance and transmittance measurements on wheat (Figs. 6 and 7). We could not find similar data for fir wood. The fluorescence spectral distribution $(\eta)$ was measured on isolated chloroplasts at room temperature by Siffel and Sestak (1988) (Fig. 8).

At run time, certain input data values are requested. Some will be discussed. First this is the fluorescence quantum efficiency $(\phi)$. A choice in between 0.02 (optimal photosynthesis) and 0.12 (blocked photosynthesis) should be made. The second input parameter is the relative chlorophyll density. The absorbtion coefficient ( $k$; see Fig. 6) is proportional to the amount of chlorophyll in the leaf. To simulate the effects of changes in the chlorophyll content, $k$ is corrected by multiplica-



Figure 7. Spectral distribution of the KM scattering coefficient $(s)$ as used in the KMF model.

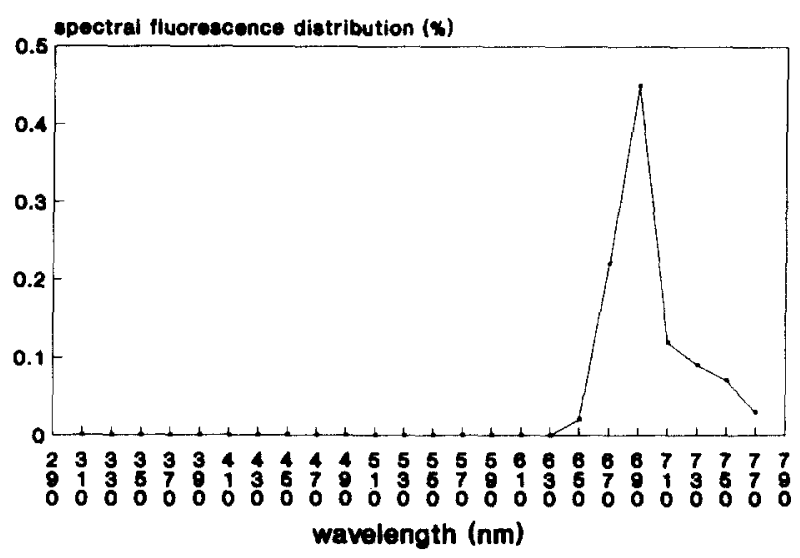

Figure 8. Spectral distribution of fluorescence as used in the KMF model (data from Siffel and Sesták, 1988).

tion with a relative chlorophyll density, that is, relative with respect to the chlorophyll density of the reference leaf, to which $k$ applies. In this way the effect of leaf green shades and yellowing may be studied.

A third input parameter is the reflectance of the background, which is assumed grey. In the field the variation of this quantity is not very large. In a laboratory situation, however, backgrounds may vary between black and white. Particularly in the near infrared, that is, beyond $710 \mathrm{~nm}$, this may have a considerable effect on the fluorescent flux.

The last parameter for the KMF leaf model is the leaf stack number. In this way we can simulate the effect of multiple leafs or, which is equivalent, the effect of a reduced or increased leaf thickness. In the canopy model, there are two additional parameters to be specified: the leaf area index (LAI) and a (predefined) leaf inclination distribution function (LIDF). 
The basic model output consists of the reflectance and fluorescence, specified for $20 \mathrm{~nm}$ wide spectral bands, and expressed as an energetic fraction of the incident excitation light.

In addition some other parameters may be generated that may be useful for practical application, for example, the fluorescence ratio (FR):

$$
\mathrm{FR}=F_{690} / F_{730} .
$$

Here $F_{690}$ is the fluorescence in the wavelength band of 680-700 nm. According to Lichtenthaler and Rinderle (1988), this ratio is related to chlorophyll content. Another potentially interesting parameter is the passive fluorescence index (FI), originally proposed by Rosema and Werner (1983) as

$$
\mathrm{FI}=\left(R_{690}-R_{650}\right) / R_{650},
$$

where $R$ is the reflection (including possible fluorescence). Finally we may extract a "vegetation index" (VI) from the reflection values, which could serve as an estimator of canopy cover or leaf area, for example,

$$
\mathrm{VI}=\left(R_{730}-R_{650}\right) /\left(R_{730}+R_{650}\right) .
$$

\subsection{Model Sensitivity}

The ultimate objective of our work is to extract photosynthesis information from the fluorescence measurements with the LEAF instrument, which measures fluorescence in two $20 \mathrm{~nm}$ wide bands, centered at $685 \mathrm{~nm}$ and $730 \mathrm{~nm}$. A model sensitivity study may give a first insight in the relative magnitude of the effects of the various canopy variables on the fluorescence signal at these two wavelengths. It will show which variables have a significant influence and which variables may possibly be neglected. Since canopy light interaction is different at both wavelength bands, a sensitivity study may also indicate which band is to be preferred, or how the two bands might be used in combination. In the following subsections we will briefly discuss the influence of various parameters, on both the leaf and the canopy fluorescence.

To study the sensitivity, a reference situation is simulated first and then each time an input parameter is varied. The reference input for the KMF leaf model and the FLSAIL canopy model is

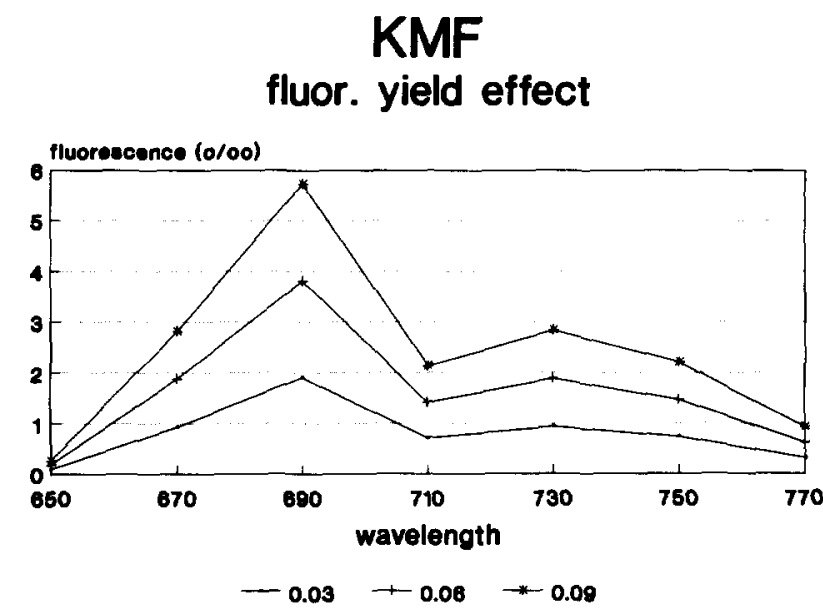

Figure 9. Effect of fluorescence yield on leaf fluorescence.

\section{FLSAIL \\ fluor. yield effect}

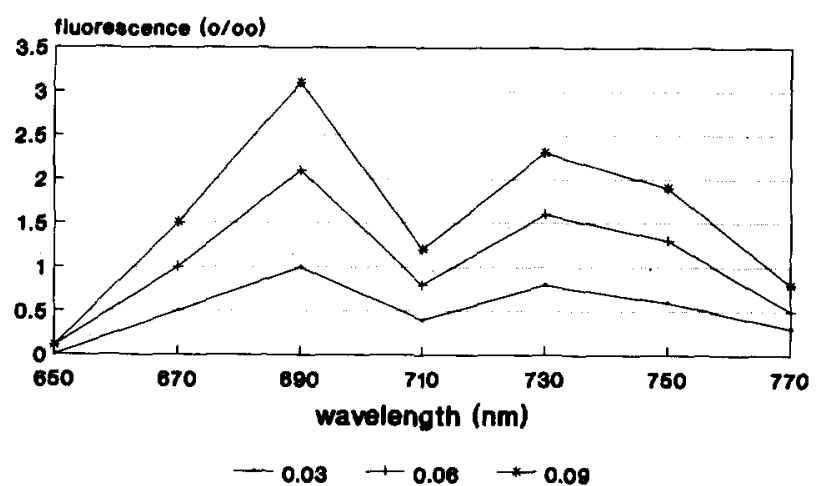

Figure 10. Effect of fluores-

\begin{tabular}{|c|c|c|}
\hline & $\underline{\mathrm{KMF}}$ & FLSAIL \\
\hline Fluorescence yield & 0.06 & 0.06 \\
\hline Excitation wavelength & $530 \mathrm{~nm}$ & $530 \mathrm{~nm}$ \\
\hline Leaf stack number, & & \\
\hline $\begin{array}{l}\text { LAI resp. } \\
\text { Relative chlorophyll }\end{array}$ & 1 & 3 \\
\hline density & 1 & 1 \\
\hline Background reflection & 0 & 0 \\
\hline Leaf angle distribution & & spherical \\
\hline $\begin{array}{l}\text { Excitation source } \\
\text { zenith angle }\end{array}$ & 0 & 0 \\
\hline Viewing zenith ang & 0 & 0 \\
\hline Relative azimuth angl & 0 & 0 \\
\hline
\end{tabular}

cence yield on canopy fluorescence.

chosen as follows:

The simulated fluorescence spectra are shown in Figures 9-19. The fluorescence is expressed as a permillage of the excitation beam energy. 


\section{KMF \\ exit. wavelength effect}

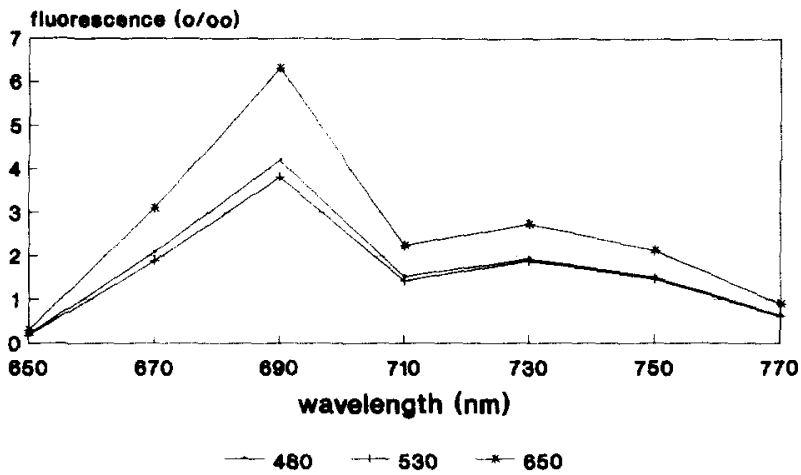

Figure 11. Effect of excitation wavelength on leaf fluores cence.

\section{FLSAIL}

exit. wavelength effect

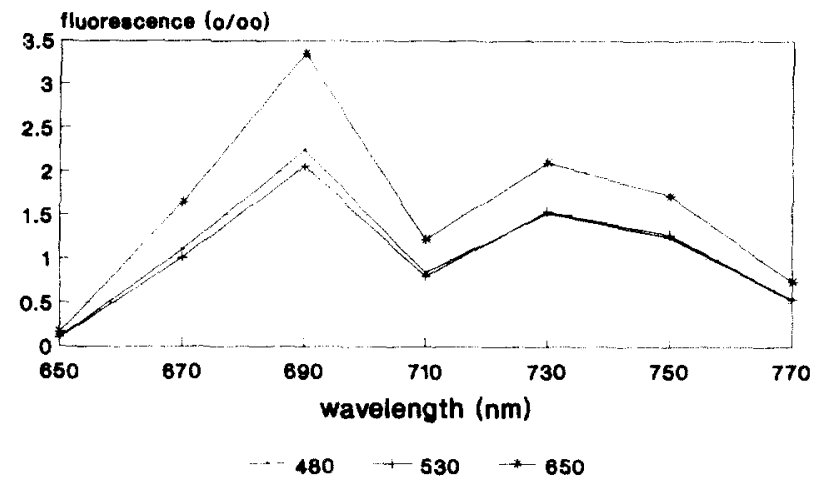

Figure 12. Effect of excitation wavelength on canopy fluorescence.

\section{Fluorescence Yield}

The effect of changes in the quantum yield on the leaf and canopy fluorescence is, of course, proportional. Figures 9 and 10 show the simulation results with the KMF leaf model and the FLSAIL crop model. The reference canopy with $\mathrm{LAI}=3$ has a lower fluorescence than the single leaf, which is likely caused by some direct background view.

\section{Excitation Wavelength}

Simulations have been carried out for excitation at $480 \mathrm{~nm}, 530 \mathrm{~nm}$, and $650 \mathrm{~nm}$. The $480 \mathrm{~nm}$ wavelength (blue) is used in the FLIDAR system of IROE/CNR, Florence, which has been used in the LIFT project. The $530 \mathrm{~nm}$ wavelength corresponds to the ND:Yag laser, which will be used in the LEAF laser fluorosensor. The $650 \mathrm{~nm}$ one (red

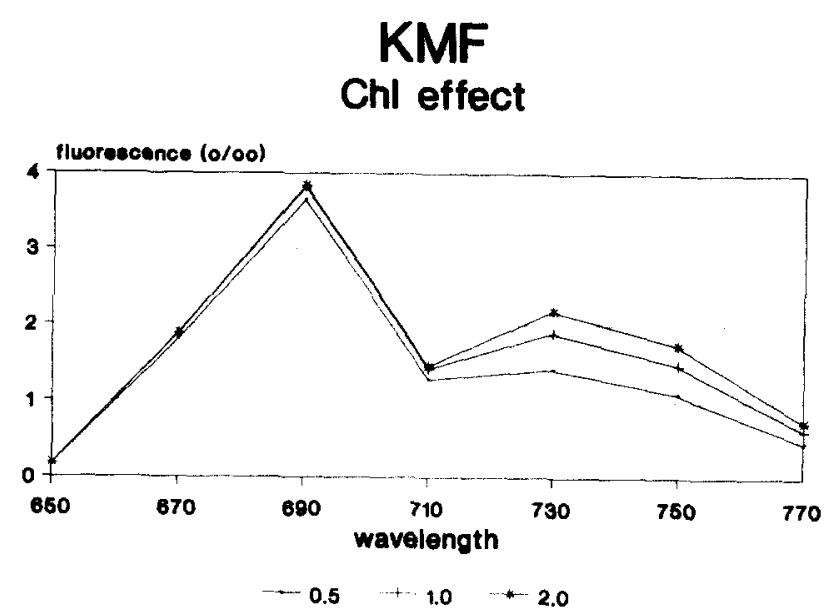

Figure 13. Effect of relative chlorophyll amount on leaf fluorescence.

\section{FLSAIL Chl effect}

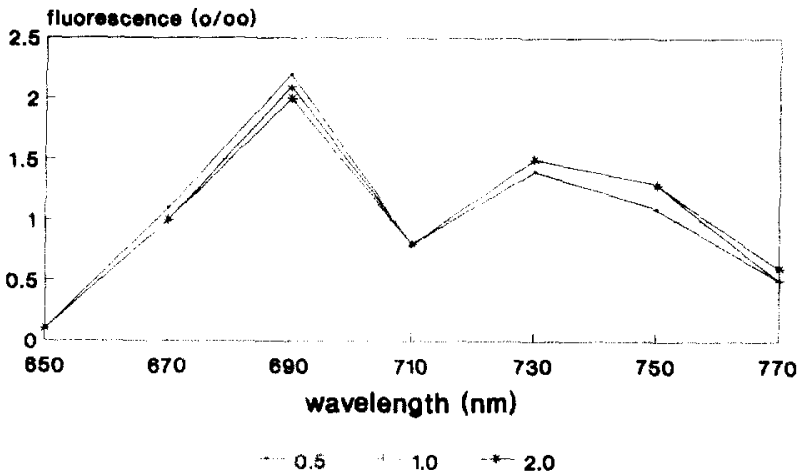

Figure 14. Effect of relitive chlorophyll amount on canopy fluorescence.

led) is applied in many laboratory fluorometers. From Figures 11 and 12 it is clear that $650 \mathrm{~nm}$ excitation gives the highest fluorescence energy vield. For blue and green excitation there is very little difference.

\section{Chlorophyll Amount}

Chlorophyll content has been doubled and halved (Figs. 13 and 14). The effects are remarkably small. Particularly the canopy fluorescence shows very little change. Leaf fluorescence shows a notable change only beyond $710 \mathrm{~nm}$.

\section{Ground Reflection}

Simulations for two extremes, a black and a white background, are shown in Figures 15 and 16 . Below $710 \mathrm{~nm}$ the effect is negligible. At longer wavelength it is not. 
KMF

ground refl. effect

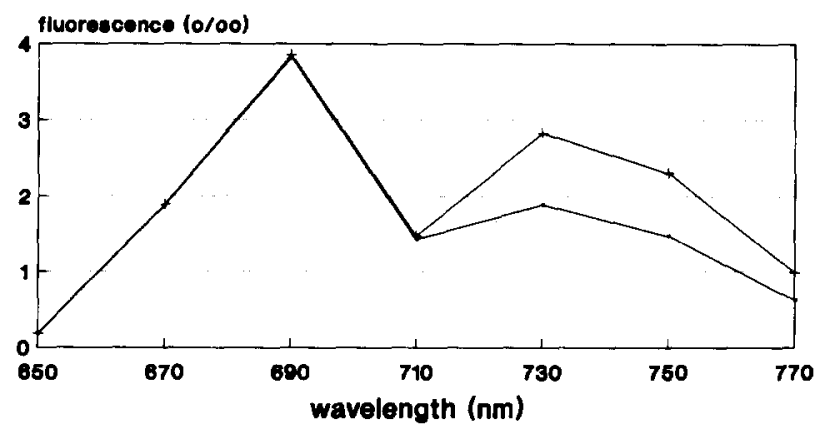

$-0.0+1.0$

Figure 15. Effect of background reflectance on leaf fluorescence.

FLSAIL ground refl. effect



$\rightarrow 0.0+1.0$

Figure 16. Effect of background reflectance on canopy fluorescence.

\section{Leaf Stack and LAI}

The simulations with the leaf model show that, when leaves are stacked, there is no effect on the fluorescence at $690 \mathrm{~nm}$ (Fig. 17). At $730 \mathrm{~nm}$, where transmission is higher, there is some increase of fluorescence, up to a number of three leaves. However, the effect of LAI on the canopy fluorescence (Fig. 18) is very significant at all fluorescence wavelengths.

\section{Leaf Orientation}

This parameter applies only to the canopy fluorescence. Simulation for three standard leaf distributions were carried out: spherical $\left(57^{\circ}\right)$, plagiophile $\left(45^{\circ}\right)$, and planophile $\left(10^{\circ}\right)$. The figures between brackets are the values of the average leaf inclination angle. The fluorescence ap-

\section{KMF \\ leaf stack number effect}



- n-1. $\quad$ - $n=3 . \quad * n=6$.

Figure 17. Effect of leaf stacking on leaf fluorescence.

\section{FLSAIL LAl effect}

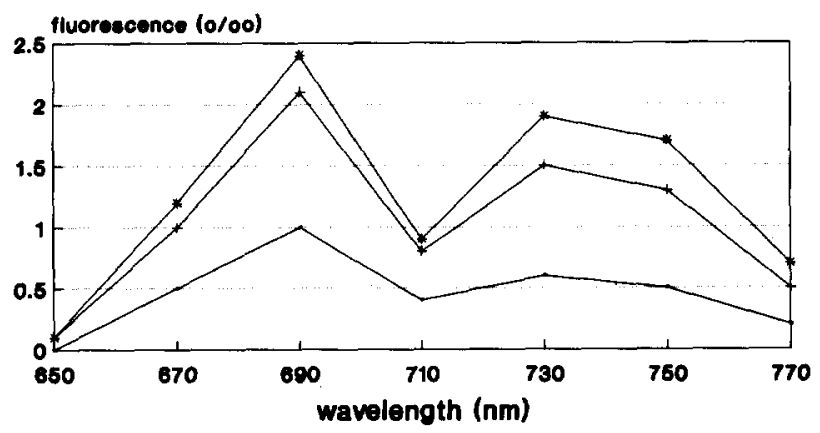

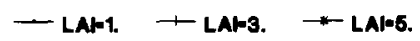

Figure 18. Effect of leaf area index on canopy fluorescence.

\section{FLSAIL leaf orientation effect}

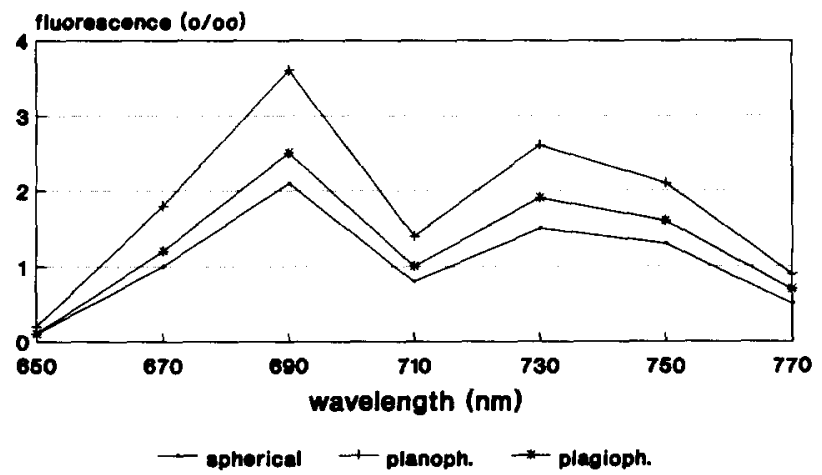

Figure 19. Effect of leaf orientation on canopy fluorescence. 
pears to be inversily proportional to the average leaf inclination (Fig. 19). The fluorescence of the planophile canopy approaches that of the single leaf, but somewhat higher at $730 \mathrm{~nm}$ and somewhat lower at $690 \mathrm{~nm}$.

\subsection{Conclusion}

From the simulation results, discussed above, it is concluded that the short wavelength fluorescence, that is, around $685 \mathrm{~nm}$, is most suitable for our goal. At this wavelength the leaf fluorescence can be considered to depend only on the fluorescence quantum yield, that is, photosynthesis. The canopy fluorescence, however, is also strongly influenced by canopy structure. Beyond $710 \mathrm{~nm}$ there is a stronger sensitivity for other parameters, such as chlorophyll content and background reflection. This would make an analysis in terms of photosynthesis and stress more problematic.

The main question to be solved in the near future is how to eliminate the canopy structure effect from the LEAF $685 \mathrm{~nm}$ fluorescence signal. This requires additional information, which can possibly be derived from the passive measurement of the LEAF system. We are presently investigating this possibility and our findings will be the subject of a future publication.

\section{APPENDIX: SOLVING BY MEANS OF THE “DOUBLING METHOD”}

The "doubling method" is based on the consideration that the optical properties of the actual leaf can be derived from the properties of a very thin leaf layer, by doubling this layer in a number of steps. Between the single and the double layer, recurrence formulae apply that are repeated for each doubling step, until the actual leaf thickness is reached.

Consider a thin leaf layer, without background. Between the incident, reflected, and transmitted radiation, the following relations may be defined:

$$
\begin{aligned}
& F^{+}(0)=r \cdot F^{-}(0)+g \cdot E^{-}(0), \\
& F^{-}(1)=t \cdot F^{-}(0)+f \cdot E^{-}(0), \\
& E^{+}(0)=r^{\prime} \cdot E^{-}(0), \\
& E^{-}(1)=t^{\prime} \cdot E^{-}(0) .
\end{aligned}
$$

\section{Exitation Fluorescence}

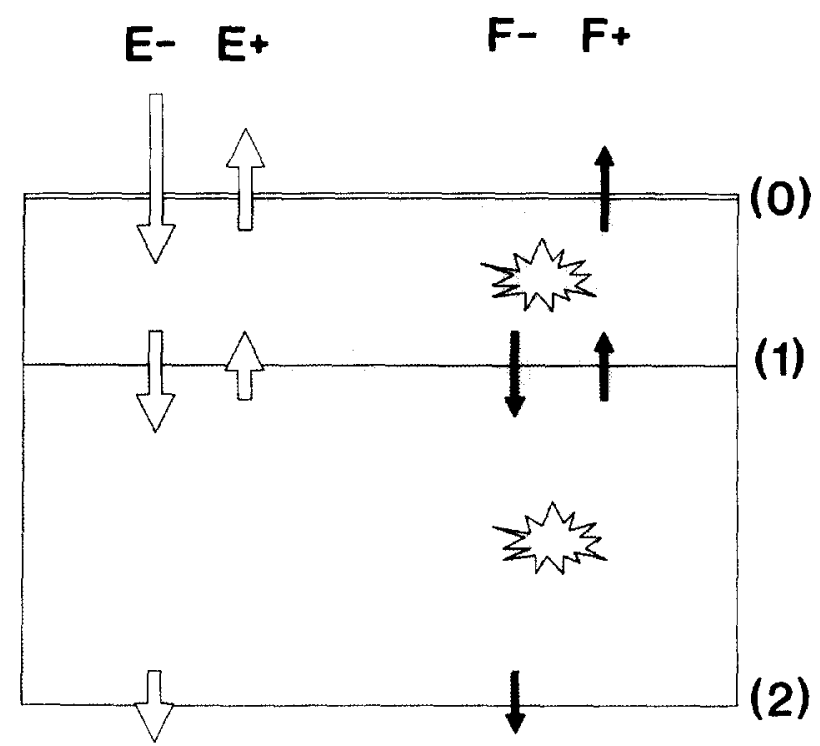

Figure 20. Sketch of the two-layer system of the doubling method.

The meaning of the various fluxes is depicted in Figure 20. In addition, it is noted that distinction is made between the fluorescent flux (e.g., $F^{-}$) and the excitation flux (e.g., $E^{-}$). $r, r^{\prime}$ and $t, t^{\prime}$ are the layer reflectance and transmittance at fluorescence and excitation wavelength, respectively. $g$ and $f$ are the front and back side fluorescent response to the incident excitation flux. If $k$ is the absorption coefficient and $s$ the backscatter coefficient, then in the case of an optically very thin layer, the various coefficients in the above equations are as follows:

$$
\begin{aligned}
r & =s \cdot d z, \\
r^{\prime} & =s^{\prime} \cdot d z, \\
t & =1-(k+s) \cdot d z, \\
t^{\prime} & =1-\left(k^{\prime}+s^{\prime}\right) \cdot d z, \\
g & =f=\phi \eta k^{\prime}\left(\lambda^{\prime} / \lambda\right) \cdot d z / 2 .
\end{aligned}
$$

If we now add a second identical thin layer, the following relations between the down- and upward fluxes may be derived. For the fluorescent fluxes,

$$
\begin{aligned}
F^{+}(0)= & r \cdot F^{-1}(0)+t \cdot F^{+}(1) \\
& +g \cdot E^{-}(0)+f \cdot E^{+}(1), \\
F^{-}(1)= & t \cdot F^{-}(0)+r \cdot F^{+}(1) \\
& +f \cdot E^{-}(0)+g \cdot E^{+}(1), \\
F^{+}(1)= & r \cdot F^{-}(1)+g \cdot E^{-}(1), \\
F^{-}(2)= & t \cdot F^{-}(1)+f \cdot E^{-}(1),
\end{aligned}
$$


and for the excitation fluxes,

$$
\begin{aligned}
& E^{+}(0)=r^{\prime} \cdot E^{-}(0)+t^{\prime} \cdot E^{+}(1), \\
& E^{-}(1)=t^{\prime} \cdot E^{-}(0)+R^{\prime} \cdot E^{+}(1), \\
& E^{+}(1)=r^{\prime} \cdot E^{-}(1), \\
& E^{-}(2)=t^{\prime} \cdot E^{-}(1) .
\end{aligned}
$$

Using Eqs. (18)-(25), we may express the reflected $\left[F^{+}(0), E^{+}(0)\right]$ and the transmitted $\left[F^{-}(2), E^{-}(2)\right]$ fluxes of the double layer in terms of the incident fluxes $\left[F^{-}(0)\right.$ and $\left.E^{-}(0)\right]$. For the fluorescent radiation this leads to the following result for the transmission,

$$
F^{-}(2)=t t \cdot F^{-}(0)+f f \cdot E^{-}(0),
$$

where

$$
\begin{aligned}
t t & =x \cdot t, \\
f f & =\left[f\left(x+x^{\prime}\right)+x x^{\prime} g\left(r+r^{\prime}\right)\right], \\
x & =t /(1-r \cdot r), \\
x^{\prime} & =t^{\prime} /\left(1-r^{\prime} \cdot r^{\prime}\right) .
\end{aligned}
$$

For the double layer reflection we obtain,

$$
F^{+}(0)=r r \cdot F^{-}(0)+g g \cdot E^{-}(0),
$$

where

$$
\begin{aligned}
r r & =r(1+x t), \\
g g & =\left[g\left(1+x^{\prime} t\right)+f\left(x^{\prime} r^{\prime}+x r\right)+x r x^{\prime} g\left(r+r^{\prime}\right)\right]
\end{aligned}
$$

For the excitation fluxes the results are similar but more simple, since the fluorescent emission terms do not occur ( $g=f=0$ ). Equations (26) and (31) of the double layer are similar to Eqs. (10) and (9) respectively of the monolayer. Thus (27), (28), (32), and (33) are the recurrent relations that relate the optical properties of the double layer to those of the monolayer. The next step is to redouble the double layer by substituting $r=r, g=g g$, $t=t t$, and $f=f f$, and thus calculating the optical properties of the quadruple layer, etc.

If $Z$ is the thickness of the leaf, $d Z$ the thickness of the initial thin layer, and $n$ the number of doubling steps, then they are related as follows:

$$
z / d z=2^{n} \text {. }
$$

If we assume, tentatively, that $d Z$ should be in the order of the size of a photosystem or the wavelength, that is, $10^{-7} \mathrm{~m}$, and that the thickness of a leaf is $10^{-3} \mathrm{~m}$, then we find $n=14$.
This study was enabled thanks to the financial support of the Netherlands Remote Sensing Board. We thank the members of the LEAF project group for reading and discussing the manuscript, particularly Dr. L. Mertens (Nedinsco, Venlo), Dr. O. van Kooten (ATO Agrotechnology, Wageningen), and Dr. C. Kliffen (IPO, Wageningen).

\section{REFERENCES}

Allen, W. A., and Richardson, A. J. (1968), Interaction of light with a plant canopy, J. Opt. Soc. Am. 59(10):1376-1379.

Amesz, J., and Vredenberg, W. J. (1982), Een spel van fotonen, protonen en electronen, Intermediair 18(1):25-31.

Barber, J., Horler, D. N. H., and Chapman, D J. (1982), Photosynthetic pigments and efficiency in relation to the spectral quality of absorbed light, ARC Photosynthesis Group, Dept. of Pure and Applied Biology, Imperial College, London.

Bunnik, N. J. J. (1978), The multispectral reflectance of shortwave radiation by agricultural crops in relation with their morphological and optical properties, Ph.D. thesis, Agricultural University of Wageningen, H. Veenman \& Zonen BV, Wageningen.

Castagnoli, F., Cecchi, G., Pantani, L., Pippi, I., Radicati, B., and Mazzinghi, P. (1986), A fluorescence lidar for land and sea remote sensing, IROE-CNR, Florence.

Cecchi, G., Pantani, L., Pippi, I., Magli, R., and Mazzinghi, P. (1984), Vegetation remote sensing: A new field for lidar applications, ECOOSA 84, Amsterdam, October.

Chapelle, E. W., Wood, F. M., Newcomb, W. W., and McMurtrey, J. E. (1984a), Laser induced fluorescence of green plants. 1: A technique for the remote detection of plant stress and species differentiation, Appl. Opt. 23(1):134-138

Chapelle, E. W., Wood, F. M., Newcomb, W. W., and McMurtrey, J. E. (1984b) Laser induced fluorescence of green plants. 2: LIF caused by nutrient deficiences in corn, Appl. Opt. 23(1):139-142.

Chapelle, E. W., Wood, F. M., Newcomb, W. W., and McMurtrey, J. E. (1985), Laser induced fluorescence of green plants. 3: LIF spectral signatures of five major plant types, Appl. Opt. 24(1):74-80.

Fukshansky, L., and Kazarinova, N. (1980), Extension of the Kubelka-Munk theory of light propagation in intensely scattering materials to fluorescent media, J. Opt. Soc. Am. 70(9):1101-1111.

Gehlhaar, U., Gunther, K. P., and Luther, J. (1981), Compact and highly sensitive fluorescence lidar for oceanographic measurements, Appl. Opt. 20:3318-3320.

Hickman, G. D., Hogg, J. E., Friedman, E. J., and Ghovanlou, A. H. (1972), Application of a pulsed laser for measurements of bathymetry and algal fluorescence, in Proc. 8th 
Int. Symp. Remote Sensing Environment, October, Ann Arbor, MI, Vol. 1, pp. 617-637.

Hoge, F. E., and Swift, R. N. (1980), Application of the NASA airborne oceanographic lidar to the mapping of chlorophyl and other organic pigments, in Chesapeake Bay Plume Study, Superflux 1980 (Janet W. Campbell, Ed.), NASA Conference Publication 2188.

Hoge, F. E., and Swift, R. N. (1983), Airborne dual laser excitation and mapping of phytoplankton photopigments in a Gulf Stream Warm Core Ring, Appl. Opt. 22(15): 2271-2281.

Hoge, F. E., Swift, R. N., and Yungel, J. K. (1983), Feasibility of airborne detection of laser induced fluorescence emissions from green terrestrial plants, Appl. Opt. 22(19): 2991-3000.

Holzwarth, A. R. (1988), Time resolved chlorophyll fluorescence, what kind of information on photosynthetic systems does it provide?, in Applications of Chlorophyl Fluorescence (H. K. Lichtenthaler, Ed.), Kluwer Academic, Dordrecht, The Netherlands, pp. 21-31.

Kim, H. H., van der Piepen, H., Amann, V., and Doerffer, R. (1985), An evaluation of $685 \mathrm{~nm}$ fluorescence imagery of coastal waters, ESA J. 9(1):17-27.

Kuusk, A. (1985), The hot spot effect of a uniform vegetative cover, Soc. J. Remote Sens. 3(4):645-658.

Lichtenthaler, H. K., Ed. (1988), Applications of chlorophyll fluorescence in photosynthesis research, stress physiology, hydrobiology and remote sensing, in Proceedings of the First International Chlorophyll Fluorescence Symposium, Bad Honeff, 6-8 June, Kluwer Academic, Dordrecht, The Netherlands.

Lichtenthaler, H. K., and Rinderle, U. (1988), Chlorophyll fluorescence signatures as vitality indicator in forest decline research, in Applications of Chlorophyl Fluorescence (H. K. Lichtenthaler, Ed.), Kluwer Academic, Dordrecht, The Netherlands, pp. 143-150.
MacFarlane, G., Watson, R. D., Theisen, A. F., et al. (1980), Plant stress detection by remote measurement of fluorescence, Appl. Opt. 19(19):3287-3289.

Neville, R. A., and Gower, J. F. R. (1976), Passive remote sensing of phytoplankton via chlorophyl-a fluorescence, $J$. Geophys. Res. 82(24):3487-3493.

Rosema, A., and Werner, W. (1983), An investigation of leaf fluorescence in relation to environmental pollution, Report to the Ministry of Education and Science, 20 July 1983, EARS bv, Delft, The Netherlands (in Dutch).

Rosema, A., Cechi, G., Pantani, L., et al. (1988), Results of the "LIFT" project: Air pollution effects the fluorescence of Douglas fir and poplar, in Applications of Chlorophyl Fluorescence (H. K. Lichtenthaler, Ed.), Kluwer Academic, Dordrecht, The Netherlands, pp. 307-317.

Schreiber, U., and Bilger, W. (1987), in Plant Response to Stress (J. D. Tenhunen et al., Eds.), Springer-Verlag, Berlin, Heidelberg.

Schreiber, U., Schliwa, U., and Bilger, W. (1986), Continuous recording of photochemical and non-photochemical chlorophyll fluorescence, quenching with a new type of modulation fluorometer, Photosynth. Res. 10:51-62.

Siffel, L. P., and Sesták, Z. (1988), Low temperature fluorescence spectra of chloroplasts: Methodical aspects and possible applications, in Applications of Chlorophyl Fluorescence (H. K. Lichtenthaler, Ed.), Kluwer Academic, Dordrecht, The Netherlands, pp. 55-62.

Suits, G. H. (1972), The calculation of the directional reflectance of a vegetative canopy, Remote Sens. Environ. $2: 117-125$.

Verhoef, W. (1984), Light scattering by leaf layers with application to canopy reflectance modeling; the SAIL model, Remote Sens. Environ. 16:125-141.

Verhoef, W. (1985), Earth observation modeling based on layer scattering matrices, Remote Sens. Environ. $17: 165-178$. 